\title{
Natural History of Vestibular Schwannoma Growth and Hearing Decline in Newly Diagnosed Neurofibromatosis Type 2 Patients
}

\section{Citation}

Plotkin, Scott R., Vanessa L. Merker, Alona Muzikansky, Fred G. Barker, and William Slattery. 2014. "Natural History of Vestibular Schwannoma Growth and Hearing Decline in Newly Diagnosed Neurofibromatosis Type 2 Patients." Otology \& Neurotology 35 (1) (January): e50-e56. doi:10.1097/mao.0000000000000239.

\section{Published Version}

doi:10.1097/MA0.0000000000000239

\section{Permanent link}

http://nrs.harvard.edu/urn-3:HUL.InstRepos:33788499

\section{Terms of Use}

This article was downloaded from Harvard University's DASH repository, and is made available under the terms and conditions applicable to Other Posted Material, as set forth at http:// nrs.harvard.edu/urn-3:HUL.InstRepos:dash.current.terms-of-use\#LAA

\section{Share Your Story}

The Harvard community has made this article openly available.

Please share how this access benefits you. Submit a story.

Accessibility 
Natural history of vestibular schwannoma growth and hearing decline in newly diagnosed neurofibromatosis type 2 patients

Scott R. Plotkin, MD, $\mathrm{PhD}^{1}$, Vanessa L. Merker, $\mathrm{BS}^{1}$, Alona Muzikansky, $\mathrm{MA}^{2}$, Fred G. Barker, II, $\mathrm{MD}^{3}$, William Slattery, III, $\mathrm{MD}^{4}$

1. Department of Neurology and Cancer Center, Massachusetts General Hospital, Boston, MA

2. Biostatistics Center, Massachusetts General Hospital, Boston, MA

3. Neurosurgical Service, Massachusetts General Hospital, Boston, MA

4. Neurotology, House Clinic, Los Angeles, CA

Corresponding author:

Scott R. Plotkin, MD, PhD

Yawkey 9E

Massachusetts General Hospital

55 Fruit Street

Boston, MA 02114

Telephone: 617-726-3650

Fax: 617-643-2591

Email: splotkin@partners.org

Running head: Natural history of NF2 VS

Sources of Funding: The NF2 Natural History Consortium was supported by the Department of

Defense award number DAMD 17-98-1-8612 to WS. 


\section{ABSTRACT}

Objective: To determine the rate of growth in vestibular schwannomas and the rate of hearing decline in neurofibromatosis type 2 (NF2) patients not undergoing active treatment

Study Design: Prospective study.

Setting: Data were collected at ten NF2 centers, including hospital-based, academic, and tertiary care centers.

Patients: 120 NF2 patients with 200 vestibular schwannomas

Outcome Measures: Hearing decline, defined as a decrease in word recognition score outside the $95 \%$ critical difference compared with baseline, and radiographic progression, defined as a $\geq$ $20 \%$ increase in tumor volume compared with baseline.

Results: During a total of 313.4 patient-years of follow up, the rate of hearing decline was $5 \%$ at 1 year, $13 \%$ at 2 years, and $16 \%$ at 3 years; the rate of tumor progression was $31 \%$ at 1 year, $64 \%$ at 2 years, and $79 \%$ at 3 years. For this cohort, the median time to tumor progression (14 months) was significantly shorter than the median time to hearing decline (62.0 months).

Conclusion: These data provide potentially useful information for the design of clinical trials for NF2 vestibular schwannoma. 


\section{INTRODUCTION}

Neurofibromatosis type 2 (NF2) is a dominantly inherited genetic condition in which patients typically develop bilateral vestibular schwannomas (VS), the hallmark of the disease, as well as schwannomas of other cranial nerves, meningiomas, and other less common tumors. ${ }^{1}$ Untreated patients typically lose hearing over time, and may acquire other neurological deficits because of cranial nerve and brainstem compression. Currently, standard active management of vestibular schwannomas may include surgery, radiation, or a combination of the two. However, both surgery and radiation for VS are commonly followed by loss of hearing or other neurological deficits in NF2 patients, who have worse results after these treatments than sporadic VS patients. ${ }^{2 ; 3}$

Balancing active management against observation is a challenge in NF2, and an improved understanding of the natural history of the disease would be of use to clinicians and patients. In addition, researchers are beginning to test medical treatments intended to prevent growth or cause shrinkage of VS in NF2 patients ${ }^{4-6}$, and accurate knowledge of expected tumor growth in the untreated state is a necessary part of designing clinical trials for such drugs.

Although many studies of natural history have been reported for sporadic VS, NF2 is a rare disease and natural history data are limited. Several single-institution clinical series have been reported from NF2 centers, but these have typically been small and retrospective. ${ }^{7-11}$ In addition, many of these studies have reported results primarily as the average growth rates or hearing decline among all subjects. This measure is less useful in designing clinical trials for drug treatment of NF2-related VS. For this purpose, an understanding of individual times to failure for measures such as tumor growth or hearing decline in untreated patients is required. 
Ideally, such a trial would use endpoint measures similar to those that have recently been proposed for use in NF2-related VS trials, ${ }^{12 ; 13}$ and would be large, prospective, and multicenter.

The NF2 Natural History Consortium was established in 1998 to acquire prospective observational data on the change in VS tumor size and hearing in NF2 patients not undergoing active treatment. This prospective multicenter observational study, which was funded through the US Department of Defense Congressionally Directed Medical Research program, enrolled patients at 10 centers in 6 countries between 1999 and 2001. Patients were followed using serial MRI and audiometry studies, and results were reported in previous publications. ${ }^{14-18}$ In the present study, we used the data acquired by the NF2 Natural History Consortium in an analysis intended specifically to inform design of future trials of therapy in NF2-related VS. Specifically, we aimed to define the expected rates over time of tumor growth and hearing decline using endpoints shown to be feasible in recent clinical trials for NF2-related VS.

\section{MATERIALS AND METHODS}

\section{Study Cohort}

Subjects who carried a diagnosis of NF2 according to National Institutes of Health criteria ${ }^{19}$ and who were being followed without active treatment at the study centers were recruited between 1999 and 2000 for the NF2 Natural History of Vestibular Schwannomas study. Study participants were aged 5 years or older and had at least 1 untreated VS ${ }^{17}$. A total of 141 patients were recruited for the study by the NF2 Natural History Consortium at 10 international medical centers with expertise in NF2 (see text, Supplemental Digital Content 1 for full list of centers and principal investigators). We analyzed data from 120 patients $(85 \%)$ who completed at least two 
study visits (full study cohort). Changes in hearing function and tumor size were analyzed in subsets of the full study cohort based on available data and requirements of the analysis (Fig. 1).

\section{Monitoring}

Prospective data collection included annual audiologic evaluations and MRIs ordered as part of clinical care. Audiologic evaluations included determination of pure tone thresholds and word recognition scores ${ }^{20 ; 21}$. Study MRI scans included post-contrast T1-weighted axial images acquired using a standardized acquisition protocol across all MRI facilities. ${ }^{18}$ The digitized data were sent to WorldCare Clinical, Inc, (Boston, MA) for centralized image analysis. Volumetric analysis of VS was performed as previously reported. ${ }^{18}$

\section{Criteria for hearing decline or improvement}

Following recent recommendations for hearing endpoints in NF2-related VS trials ${ }^{13}$, hearing decline was defined as a statistically significant decrease in word recognition score $(\mathrm{p}<$ 0.05) compared to the baseline either due to tumor progression or to surgery. Patients were eligible for progressive hearing decline if their baseline word recognition score was $\geq 6 \%$ on a 50-word list (i.e., allowed for a measurable decrease below the lower limit of the critical difference threshold - the "floor effect"). Published tables to determine significant differences between word recognition scores were used to compare each patient's follow-up tests to baseline values $^{22}$. Hearing improvement was defined as a statistically significant increase in word recognition score $(p<0.05)$ compared to the baseline score on at least one measurement ${ }^{23}$. Patients were eligible for spontaneous hearing improvement if their pretreatment word recognition score was $\leq 94 \%$ on a 50 -word list (i.e., if their baseline hearing allowed for a 
measurable increase above the upper limit of the critical difference threshold - "the ceiling effect"). Patients who were deafened due to prior vestibular schwannoma surgery were not considered eligible for hearing improvement.

\section{Criteria for tumor progression or shrinkage}

Radiographic progression was defined as an increase in tumor volume of greater than or equal to $20 \%$ compared to the baseline, as recommended for NF2-related VS trial endpoints. ${ }^{13}$ Survival times were calculated from the first study assessment after enrollment until the study closed in 2005. Tumor shrinkage was defined as a decrease in tumor volume of greater than or equal to $20 \%$ compared to baseline value on at least 1 measurement.

\section{Statistical Analyses}

We report descriptive statistics for clinical and demographic factors for the study cohort. Spearman correlation coefficient was used to determine relationship between baseline tumor size and baseline WRS. We used Kaplan Meier methods to estimate time to hearing decline and time to radiographic progression and Cox proportional hazards regression to test for factors predicting time to hearing decline. Because patients on clinical trials typically have larger vestibular schwannomas, we also report stratified Kaplan-Meier analyses comparing hearing outcomes in ears with baseline tumor volume of less than $1 \mathrm{ml}$ to those greater than or equal to $1 \mathrm{ml}$.

We analyzed the data in two ways. In the primary analysis, patients who experienced iatrogenic hearing decline were censored at the time of surgery (i.e., were not counted as having experienced hearing decline). This analysis was chosen because hearing-preservation surgery was performed in some patients without prior hearing decline. Similarly, patients who 
underwent surgery on a vestibular schwannoma before documented volumetric progression were censored at the time of surgery. The results of these analyses reflect the natural history of hearing decline and tumor growth in the absence of any surgical intervention.

A secondary time-to-failure analysis reflecting the experience of the cohort as they were actually managed is presented for comparison. In this analysis, patients with iatrogenic hearing decline at the time of surgery were not censored but were analyzed as having experienced hearing loss on the date of surgery. Similarly, the date of radiographic progression was defined as the date of surgery for patients who required VS surgery, even if volumetric progression had not yet been documented.

All statistical calculations were performed using SAS software (version 9.2, SAS Institute Inc, NC, USA). This study was approved by the institutional review board (IRB) of each institution in the NF2 Natural History Consortium and by the central Department of Defense IRB. All patients provided written informed consent. Reanalysis of the study data was approved by the Massachusetts General Hospital IRB.

\section{RESULTS}

\section{Study Cohort}

The baseline clinical characteristics of the 120 study patients (200 vestibular schwannomas) who completed at least two study visits are shown in Table 1. 60 men and 60 women with a median age of 26 years (range, 5 to 72 years) at enrollment had hearing studies performed. 32 patients $(27 \%)$ had a known family history of NF2. The mean length of follow up during the study was 31.3 months (range, 2.4 to 77.7 months) with a total follow-up of 313.4 patient-years. Baseline median tumor volume was $0.40 \mathrm{ml}$, and the baseline hearing ability for 
the 91 ears with both WRS and pure-tone averages available at baseline is documented as recommended by the AAO-HNS ${ }^{24}$ (see Table 2). There was no significant correlation between tumor volume and WRS in the 65 ears with concurrent baseline measurements $(p=0.21)$.

\section{Time to Hearing Decline}

A total of 54 patients (76 ears) could be evaluated for hearing decline. One patient was ineligible for hearing decline due to baseline word recognition score of $\leq 4 \%$ in both ears, and 65 patients were ineligible because word recognition score was not measured. In the primary analysis where patients with iatrogenic hearing loss were censored at the time of surgery, $33 \%$ percent of patients $(18 / 54)$ and $28 \%(21 / 76)$ of ears experienced a decline in word recognition during the observation period. Overall, the rates of hearing decline per ear were $5 \%$ at 1 year, $13 \%$ at 2 years, and $16 \%$ at 3 years. The median time to hearing decline was 62.0 months (Figure 2). In comparison, in the secondary analysis where iatrogenic hearing loss was considered an event, 39\% percent of patients (21/54) and 33\% (25/76) of ears experienced a decline in word recognition during the observation period. Overall, the rates of hearing decline per ear were $7 \%$ at 1 year, $18 \%$ at 2 years, and $22 \%$ at 3 years. The median time to hearing decline was 60.7 months. Rates of hearing decline using this analysis are shown in Table 3.

22/54 hearing patients in the study had bilateral VS and were evaluable for hearing decline in both ears. During the study period, $23 \%(5 / 22)$ of patients experienced progressive hearing decline in 1 ear and 14\% (3/22) in both ears. For these 3 patients, the difference between time to hearing decline in both ears ranged from 0 to 23 months.

To investigate whether baseline tumor volume influenced time to hearing outcomes, we used stratified Kaplan-Meier analyses to compare hearing outcomes in ears with baseline tumor 
volume of $<1 \mathrm{ml}$ (small tumors) to those of $\geq 1 \mathrm{ml}$ (medium/large tumors). In the 65 ears with data on baseline tumor volume and word recognition score, $47(72 \%)$ had tumor volume $<1 \mathrm{ml}$ and $18(28 \%)$ had tumor volume $\geq 1 \mathrm{ml}$. Log-rank test showed a significant difference in time to hearing decline with medium/large tumors having a shorter median time to hearing decline (38 months) compared with small tumors (median not reached) (Fig. 2; p<0.0001). Thus, larger tumors were associated with an increased risk of hearing decline during the observation period.

We also tested the following clinical factors as predictors of hearing decline on a pertumor basis: baseline tumor volume (as a continuous variable), baseline AAO-HNS hearing classification, and baseline word recognition score. Sixty-five ears with complete data on these clinical factors were included in the analysis. Tumor volume at baseline was correlated with time to hearing decline $(\mathrm{p}<0.001$, hazard ratio=1.7) Neither baseline AAO-HNS classification nor baseline hearing level correlated significantly with hearing decline $(p>0.05)$. Thus, baseline hearing status did not correlate with hearing decline during the observation period.

\section{Time to tumor progression}

In the primary statistical analysis, a total of 102 patients (166 ears) could be evaluated for tumor growth. 12 patients were ineligible because one or more tumor volumes could not be accurately calculated and 6 patients were ineligible because their candidate vestibular schwannomas were resected prior to follow up MRI. Seventy-six percent of patients (78/102) and $63 \%$ of ears $(105 / 166)$ experienced tumor progression during the observation period. Overall, the rates of progression per ear were $31 \%$ at 1 year, $64 \%$ at 2 years, and $79 \%$ at 3 years. The median time to tumor progression was 14.7 months (Figure 3). 
In the secondary analysis, a total of 108 patients (179 ears) could be evaluated for tumor growth. 12 patients were ineligible because one or more tumor volumes could not be accurately calculated. 81 percent of patients $(88 / 108)$ and $68 \%$ of ears $(121 / 179)$ experienced tumor progression during the observation period. Overall, the rates of progression per ear were $35 \%$ at 1 year, $66 \%$ at 2 years, and $80 \%$ at 3 years (Table 4 ). The median time to tumor progression was 13.6 months.

64 patients in the study had bilateral VS and were evaluable for tumor growth in both ears. During the study period, 39\% (25/64) experienced progressive growth in 1 ear and $42 \%$ $(27 / 64)$ in both ears. For these 27 patients, the time delay between progression events in both ears ranged from 0 months (i.e., simultaneous progression) to 36 months.

To investigate whether baseline tumor volume influenced time to tumor progression, we performed stratified Kaplan-Meier analyses comparing outcomes in 118 ears with baseline tumor volume of $<1 \mathrm{ml}(71 \%)$ vs. 48 with baseline tumor volume $\geq 1 \mathrm{ml}(29 \%)$ (Figure 3$)$. Log-rank tests did not show a significant difference in time to progression $(p=0.51)$. Median time to tumor progression was 14.7 months for smaller tumors and 14.5 months for larger tumors. Thus, baseline tumor volume did not predict future vestibular schwannoma tumor progression.

\section{Spontaneous hearing improvement and tumor shrinkage}

A total of 28 patients (36 ears) could be evaluated for hearing improvement. Forty-five ears could not be evaluated due to the ceiling effect of word recognition score measurements. $36 \%(13 / 36)$ of ears experienced a spontaneous improvement in word recognition score over baseline. However, improvement was present at last study follow-up for only 4/13 patients, and in all 4 of these cases, the improvement was documented only at the last visit, so durability of 
improvement was not confirmed. Additionally, 7/13 improvements were in ears with near normal hearing (i.e., baseline word recognition scores of 94\%). When only patients with baseline WRS $<90 \%$ were included, spontaneous hearing improvement occurred in 3/19 (16\%).

A total of 102 patients (166 ears) could be evaluated for spontaneous tumor shrinkage. $25 \%$ of patients (26/102) and 19\% (31/166) of VS experienced a decrease in tumor volume of $\geq 20 \%$ during the study period. However, $11 / 31$ patients did not maintain tumor shrinkage, and 8/31 patients demonstrated tumor shrinkage only at their last study visit, so durability of response could not be determined. In addition, most tumor shrinkage (24/31) occurred in small tumors (volumes $<1 \mathrm{ml}$ ). The spontaneous shrinkage rate in medium/large tumors was $15 \%$ (7/48) with a mean change in volume from $4.01 \mathrm{ml}$ to $1.91 \mathrm{ml}$; the spontaneous shrinkage rate in small tumors was $20 \%(24 / 118)$ with a mean change in volume from $0.28 \mathrm{ml}$ to $0.14 \mathrm{ml}$.

\section{DISCUSSION}

In this study, we re-analyzed hearing and imaging data on 120 patients (179 VS) collected by the NF2 Natural History Consortium to better understand the natural history of untreated NF2 vestibular schwannomas. Our goal was to inform the design of future trials of therapy by defining the expected rates over time of tumor growth and hearing decline without active treatment.

\section{Trial design: hearing and radiographic response as primary endpoints}

To date, trials for NF2-related VS have focused on tumor shrinkage or hearing improvement as primary endpoints, since these outcomes are thought to be rare in untreated tumors. In our study, the rate of spontaneous tumor shrinkage by volumetric analysis was $19 \%$. 
This rate is within the range previously reported for sporadic VS ${ }^{25}$. By documenting the spontaneous improvement rate for VS in the NF2 Natural History Study, our current data help clarify the null hypothesis for future trials. Our data suggests that such trials should focus on VS larger than $1 \mathrm{ml}$ (since small VS are more subject to variability in volumetric measurement) and with baseline WRS $\leq 90 \%$ (since apparent improvement is more common in patients with near normal hearing) in order to minimize the false positive rate (i.e., spontaneous tumor shrinkage or improvement in hearing),

Using our results, we can estimate the number of patients that would be required for a traditional phase 2 trial comparing drug treatment vs. placebo/usual care to establish superiority of the experimental arm. Supplemental digital content 2 lists trial sizes based on a radiographic or hearing response rate of $15 \%$ in the control arm. A $15 \%$ response rate was used since only $15 \%$ of tumors with volume $\geq 1 \mathrm{ml}$ experienced spontaneous tumor shrinkage (defined as decrease in volume $\geq 20 \%$ ), and similarly, hearing improvement (defined as a statistically significant increase in word recognition score) was noted in $16 \%$ of ears with WRS $<90 \%$. Figures for these subsets of patients may be more useful for modeling clinical trial data, as the uncertainty of measuring hearing improvement in VS with WRS $>90 \%{ }^{13}$ and the increased variability in volumetric measurement of small VS ${ }^{26}$ have been used as the rationale for defining a minimum tumor size and maximum hearing level for entry into NF2 clinical trials.

Given the rarity of NF2 (disease prevalence 1 in $56,000{ }^{27}$ ) and the potentially large number of agents deserving study, completing accrual for randomized clinical trials is likely to pose a challenge. Although a single arm design with historical control is inferior to a randomized study, this design can be appropriate for rare diseases where patient numbers are limited (e.g., everolimus for subependymal giant cell astrocytoma and romidepsin for cutaneous 
T-cell lymphoma). In a single arm study with historical control, drug activity for NF2 vestibular schwannoma would be suggested if the lower limit of the $95 \%$ confidence interval of the response rate to the experimental agent were greater than $15 \%$.

\section{Trial design: time to hearing decline or tumor progression as primary endpoints}

To date, clinical trials with time to hearing decline or tumor progression as the primary endpoint have not been used for NF2 vestibular schwannoma. However, trials with a primary goal of delaying hearing decline or tumor progression are desirable for NF2 patients, who are typically develop progressive morbidity related to growth of their bilateral vestibular schwannomas after initial diagnosis. Our natural history data can be used to calculate needed sample sizes for randomized phase 2 prevention studies comparing an intervention to placebo. A table of trial size using time to tumor progression or hearing decline data is shown in Supplemental Digital Content 3.

Since the median time to tumor growth $(14.7$ months $)$ in our study was shorter than the median time to hearing decline (62 months), trials focused on preventing tumor growth would usually require fewer patients to achieve the needed statistical power. Thus, trials using tumor size as a primary endpoint may be an efficient approach for identifying active drugs in the near future. For trials focused on prevention of hearing decline, we found that patients in our cohort with medium/large tumors $(\geq 1 \mathrm{ml}$ ) at study enrollment had a shorter median time to hearing decline (38 months) than small tumors (median not reached), and thus represent a more feasible group to study in clinical trials. While rate of tumor growth is not correlated to hearing preservation, $^{11}$ our results showed a correlation between larger baseline tumor size and subsequent hearing loss. For this reason, we propose that NF2 patients with VS $\geq 1 \mathrm{ml}$ be 
stratified as "high risk" for subsequent hearing decline, and that consideration be given to making these patients eligible for trials without the need for documented tumor progression at study enrollment. Emerging techniques such as radiographic identification of elevated intralabrynthine protein ${ }^{28}$ may suggest new strategies to further supplement selection of patients at highest risk for hearing loss.

As with trials focused on hearing improvement or tumor shrinkage, our natural history results can be used as a historical control for single arm studies that use time to tumor progression or hearing decline as the primary endpoint. However, this data should be used with caution as the trial cohort would have to be similar to patients enrolled in the Natural History Study (Table 1). Given the changes in diagnosis and clinical management of NF2 patients over the past 2 decades, it is not clear that current trial groups would be well matched, raising concerns for the validity of any subsequent conclusions.

Another important consideration is that current trials for NF2 vestibular schwannoma have required patients to have tumor progression at enrollment. In contrast, evidence of tumor progression was not required for participation in the Natural History Study, and many patients were clinically stable at enrollment. For this reason, the progression rates in the NF2 Natural History Study likely represent a lower estimate of progression rates than would be found without treatment in patients entering current clinical trials. We note that the observed rates of hearing decline and tumor progression in medically treated patients in current reports ${ }^{5 ; 6}$ are higher than those we found in the Natural History Study. Finally, we should note the limitations of imaging and audiology performed across many years and multiple institutions. Not all centers performed testing of word recognition score and not all evaluations were performed at the predefined 
intervals. It is possible that these variations may have introduced unforeseen bias into the study results.

The National Institutes of Health is currently enrolling patients in a new NF2 Natural History study (NCT00598351). The results from this study will help extend the findings from the completed NF2 Natural Study reported here, although from a single center rather than the multiple centers reported here. In the future, it will be helpful to have high quality data on the natural history of NF2 vestibular schwannomas after documented progression since these tumors are more likely to require treatment. In addition, future studies aimed at capturing the natural history of NF2 meningioma, non-vestibular schwannoma, and ependymoma would be helpful to guide future clinical trials in these conditions. 


\section{Reference List}

1. Asthagiri AR, Parry DM, Butman JA et al. Neurofibromatosis type 2. Lancet 2009;373:1974-1986.

2. Mathieu D, Kondziolka D, Flickinger JC et al. Stereotactic radiosurgery for vestibular schwannomas in patients with neurofibromatosis type 2: an analysis of tumor control, complications, and hearing preservation rates. Neurosurgery 2007;60:460-468.

3. Samii M, Matthies C, Tatagiba M. Management of vestibular schwannomas (acoustic neuromas): auditory and facial nerve function after resection of 120 vestibular schwannomas in patients with neurofibromatosis 2. Neurosurgery 1997;40:696-705.

4. Karajannis MA, Legault G, Hagiwara M et al. Phase II trial of lapatinib in adult and pediatric patients with neurofibromatosis type 2 and progressive vestibular schwannomas. Neuro Oncol 2012;14:1163-1170.

5. Plotkin SR, Halpin C, McKenna MJ et al. Erlotinib for progressive vestibular schwannoma in neurofibromatosis 2 patients. Otol Neurotol 2010;31:1135-1143.

6. Plotkin SR, Merker VL, Halpin C et al. Bevacizumab for progressive vestibular schwannoma in neurofibromatosis type 2: a retrospective review of 31 patients. Otol Neurotol 2012;33:1046-1052.

7. Baser ME, Makariou EV, Parry DM. Predictors of vestibular schwannoma growth in patients with neurofibromatosis Type 2. J Neurosurg 2002;96:217-222.

8. Dirks MS, Butman JA, Kim HJ et al. Long-term natural history of neurofibromatosis Type 2-associated intracranial tumors. J Neurosurg 2012;117:109-117.

9. Ito E, Saito K, Yatsuya $\mathrm{H}$ et al. Factors predicting growth of vestibular schwannoma in neurofibromatosis type 2. Neurosurg Rev 2009;32:425-433.

10. Mautner VF, Baser ME, Thakkar SD et al. Vestibular schwannoma growth in patients with neurofibromatosis Type 2: a longitudinal study. J Neurosurg 2002;96:223-228.

11. Peyre M, Goutagny S, Bah A et al. Conservative management of bilateral vestibular schwannomas in neurofibromatosis type 2 patients: hearing and tumor growth results. Neurosurgery 2013;72:907-913.

12. Blakeley JO, Evans DG, Adler J et al. Consensus recommendations for current treatments and accelerating clinical trials for patients with neurofibromatosis type 2. Am J Med Genet A 2011.

13. Plotkin SR, Halpin C, Blakeley JO et al. Suggested response criteria for phase II antitumor drug studies for neurofibromatosis type 2 related vestibular schwannoma. J Neurooncol 2009;93:61-77. 
14. Fisher LM, Doherty JK, Lev MH et al. Distribution of nonvestibular cranial nerve schwannomas in neurofibromatosis 2. Otol Neurotol 2007;28:1083-1090.

15. Fisher LM, Doherty JK, Lev MH et al. Concordance of bilateral vestibular schwannoma growth and hearing changes in neurofibromatosis 2: neurofibromatosis 2 natural history consortium. Otol Neurotol 2009;30:835-841.

16. Masuda A, Fisher LM, Oppenheimer ML et al. Hearing changes after diagnosis in neurofibromatosis type 2. Otol Neurotol 2004;25:150-154.

17. Slattery WH, III, Fisher LM, Iqbal Z et al. Vestibular schwannoma growth rates in neurofibromatosis type 2 natural history consortium subjects. Otol Neurotol 2004;25:811817.

18. Slattery WH, Lev MH, Fisher LM et al. MRI evaluation of neurofibromatosis 2 patients: a standardized approach for accuracy in interpretation. Otol Neurotol 2005;26:733-740.

19. NIH Consensus Conference. Neurofibromatosis. Conference statement. National Institutes of Health Consensus Development Conference. Arch Neurol 1988;45:575-578.

20. Monsell EM. New and revised reporting guidelines from the Committee on Hearing and Equilibrium. American Academy of Otolaryngology-Head and Neck Surgery Foundation, Inc. Otolaryngol Head Neck Surg 1995;113:176-178.

21. Hirsch IJ, DAVIS H, SILVERMAN SR et al. Development of materials for speech audiometry. J Speech Hear Disord 1952;17:321-337.

22. Halpin C, Rauch SD. Using audiometric thresholds and word recognition in a treatment study. Otol Neurotol 2006;27:110-116.

23. Thornton AR, Raffin MJ. Speech-discrimination scores modeled as a binomial variable. $J$ Speech Hear Res 1978;21:507-518.

24. Gurgel RK, Jackler RK, Dobie RA et al. A new standardized format for reporting hearing outcome in clinical trials. Otolaryngol Head Neck Surg 2012;147:803-807.

25. Huang X, Caye-Thomasen P, Stangerup SE. Distinct spontaneous shrinkage of a sporadic vestibular schwannoma. Auris Nasus Larynx 2013;40:243-246.

26. Harris GJ, Plotkin SR, MacCollin M et al. Three-dimensional volumetrics for tracking vestibular schwannoma growth in neurofibromatosis type II. Neurosurgery 2008;62:13141319.

27. Evans DG, Howard E, Giblin C et al. Birth incidence and prevalence of tumor-prone syndromes: estimates from a UK family genetic register service. Am J Med Genet A 2010;152A:327-332. 
28. Asthagiri AR, Vasquez RA, Butman JA et al. Mechanisms of hearing loss in neurofibromatosis type 2. PLoS One 2012;7:e46132. 
Table 1. Baseline characteristics of the patients.

\begin{tabular}{|c|c|c|}
\hline & All Study Patients $(n=120)$ & Patients with VS $\geq 1 \mathrm{cc}(\mathrm{n}=45)$ \\
\hline Gender (\% male) & $60(50 \%)$ & $23(51 \%)$ \\
\hline Familial Disease & $32(27 \%)$ & $11(24 \%)$ \\
\hline Age at enrollment (median) & 26 years (range, $5-72$ ) & 29 years (range, $5-72$ ) \\
\hline Age $<18$ & $31(26 \%)$ & $11(24 \%)$ \\
\hline Age $\geq 18$ & $89(74 \%)$ & $34(76 \%)$ \\
\hline Age at diagnosis (median) & 23 years & 25 years \\
\hline Length of follow-up (mean/median) & $\begin{array}{c}31.3 \text { months } / 23.3 \text { months } \\
\text { (range, } 2-78 \text { months) }\end{array}$ & $\begin{array}{c}33.4 \text { months } / 22.7 \text { months } \\
\text { (range, } 8-78 \text { months) }\end{array}$ \\
\hline \multicolumn{3}{|l|}{ Baseline VS Volume } \\
\hline Number of ears & 179 & $52^{\mathrm{a}}$ \\
\hline Median (range) & $0.40 \mathrm{cc}(0.01-47.36 \mathrm{cc})$ & $3.01 \mathrm{cc}(1.00-47.36 \mathrm{cc})$ \\
\hline$<1$ cc (small tumors) & $125(70 \%)$ & ---- \\
\hline$\geq 1$ cc (medium/large tumors) & $54(30 \%)$ & ---- \\
\hline
\end{tabular}

${ }^{\mathrm{a}}$ Of the 52 ears $\geq 1 \mathrm{cc}, 34$ ears were eligible for tumor progression analysis only, 14 ears were eligible for both tumor volume progression and hearing decline analyses, and 4 ears were eligible for hearing decline analysis only. 
Table 2. Baseline WRS and PTA for 91 ears in which both baseline values were available.

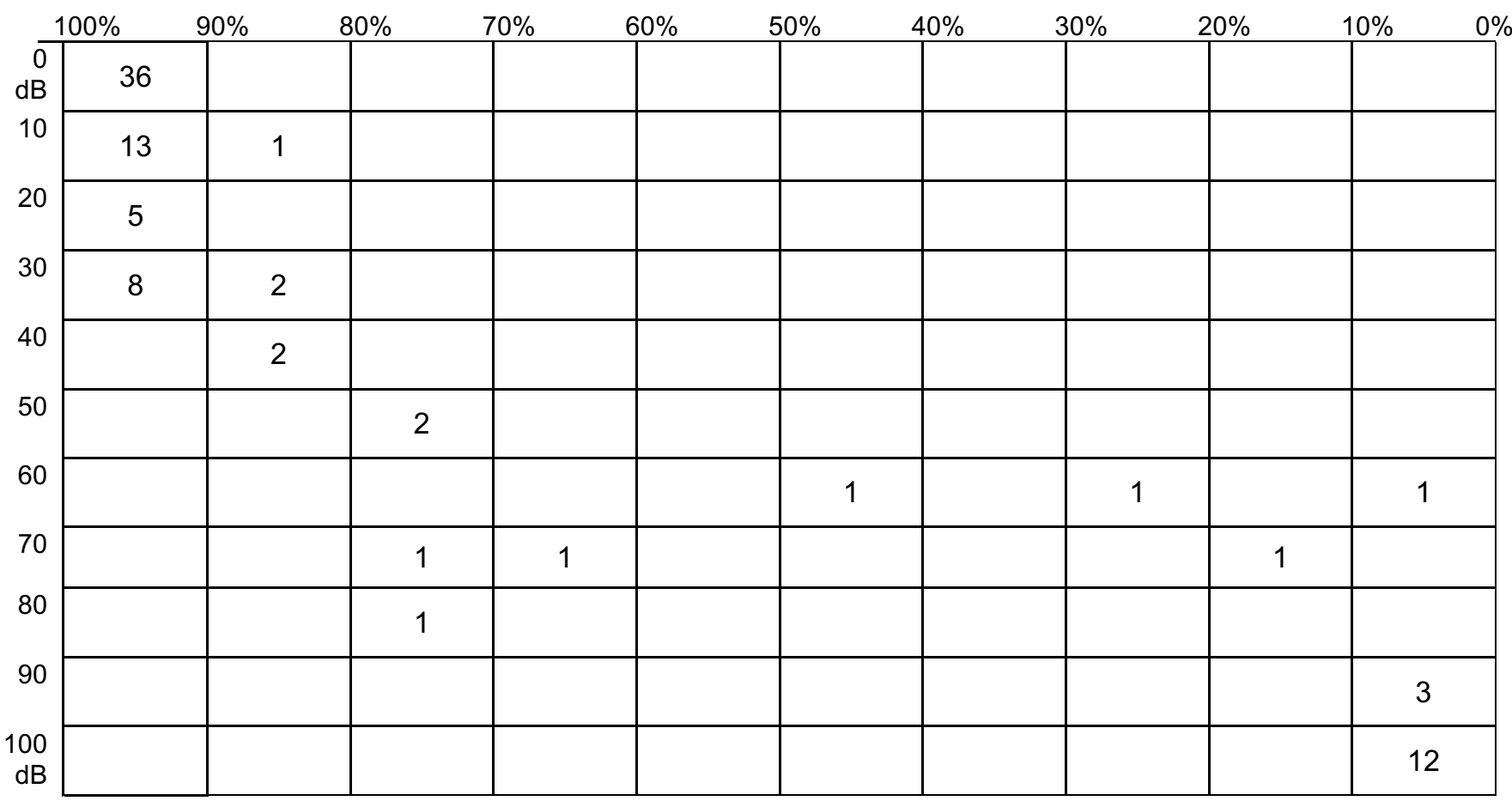


Table 3. Comparison of time to hearing decline estimates using two different censoring criteria

\begin{tabular}{|c|c|c|}
\hline & $\begin{array}{c}\text { Hearing Loss } \\
\text { (iatrogenic censored) } \\
\mathrm{N}=76 \text { ears }\end{array}$ & $\begin{array}{c}\text { Hearing Loss } \\
\text { (including iatrogenic) } \\
\mathrm{N}=76 \text { ears }\end{array}$ \\
\hline Median time to hearing decline & 62.0 months & 60.7 months \\
\hline Hearing decline at 1 year & $5 \%$ & $7 \%$ \\
\hline Hearing decline at 2 years & $13 \%$ & $18 \%$ \\
\hline Hearing decline at 3 years & $16 \%$ & $22 \%$ \\
\hline
\end{tabular}


Table 4. Comparison of time to tumor progression estimates using two different censoring criteria

\begin{tabular}{|c|c|c|}
\hline & $\begin{array}{c}\text { Radiographic progression } \\
\text { only } \\
\mathrm{N}=166 \text { ears }\end{array}$ & $\begin{array}{c}\text { Radiographic progression or } \\
\text { VS surgery } \\
\mathrm{N}=179 \text { ears }\end{array}$ \\
\hline Median time to progression & 14.7 months & 13.6 months \\
\hline Progression at 1 year & $31 \%$ & $35 \%$ \\
\hline Progression at 2 years & $64 \%$ & $66 \%$ \\
\hline Progression at 3 years & $79 \%$ & $80 \%$ \\
\hline
\end{tabular}


$\underline{\text { Figure Titles and Legends }}$

\section{Figure 1.}

Flow diagram of hearing and tumor analyses.

Figure 2

Kaplan Meier analysis of hearing decline for all vestibular schwannomas (black), small tumors ( $<1 \mathrm{ml}$, red), and medium/large tumors $(\geq 1 \mathrm{ml}$, blue). Log-rank test showed a significant difference in time to hearing decline with medium/large tumors having a shorter median time to hearing decline (38 months) than small tumors (median not reached) $(\mathrm{p}<0.0001)$. Note: 11 ears in the overall analysis were excluded from the stratified analysis (smaller vs. larger tumors) due to lack of concurrent baseline data on hearing and tumor size.

\section{Figure 3}

Kaplan Meier analysis of tumor growth for all vestibular schwannomas (black), small tumors ( $<1 \mathrm{ml}$, red), and medium/large tumors $(\geq 1 \mathrm{ml}$, blue). Log-rank test showed no significant difference in time to tumor growth between smaller and larger tumors. 


\section{Figure 1}

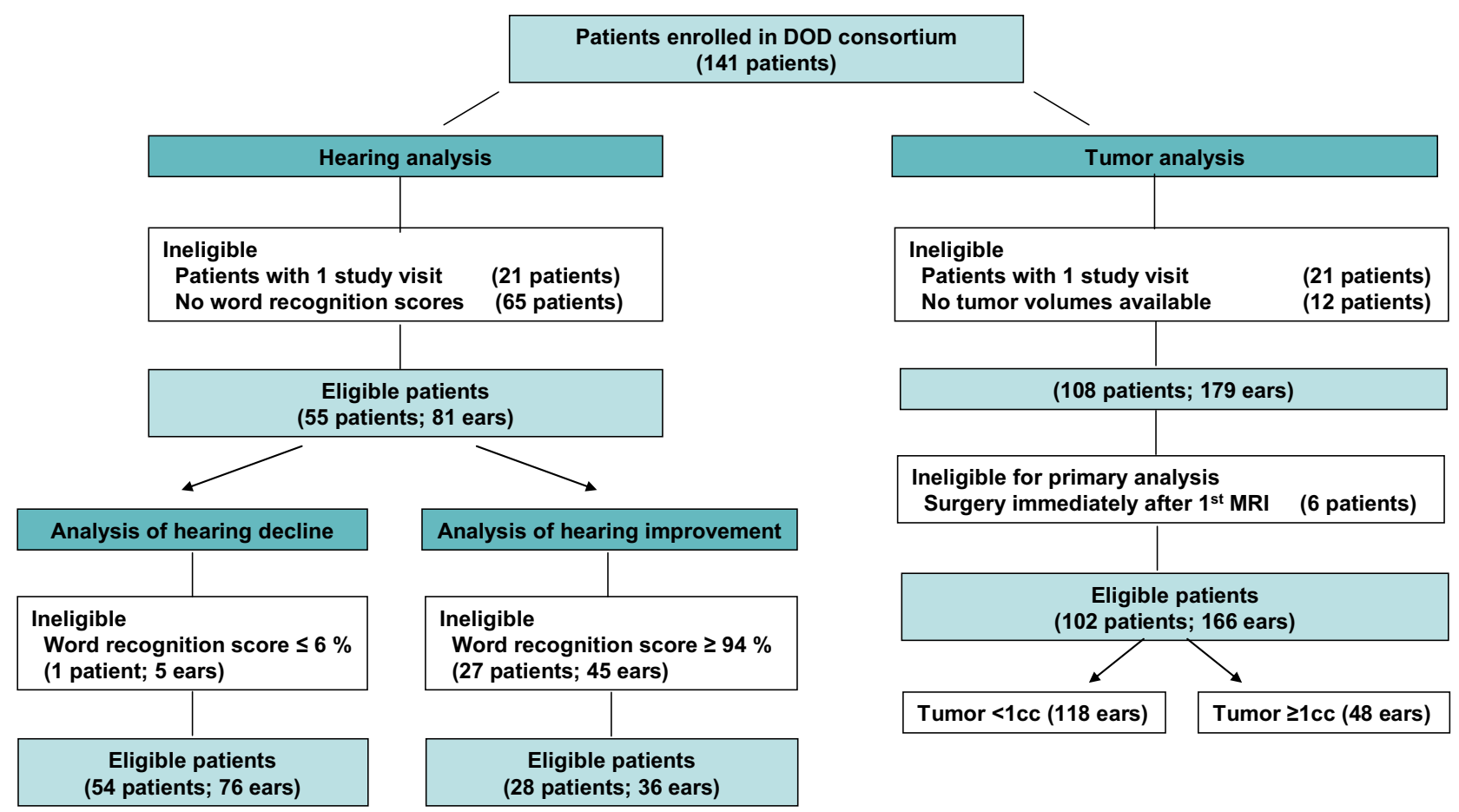


Figure 2

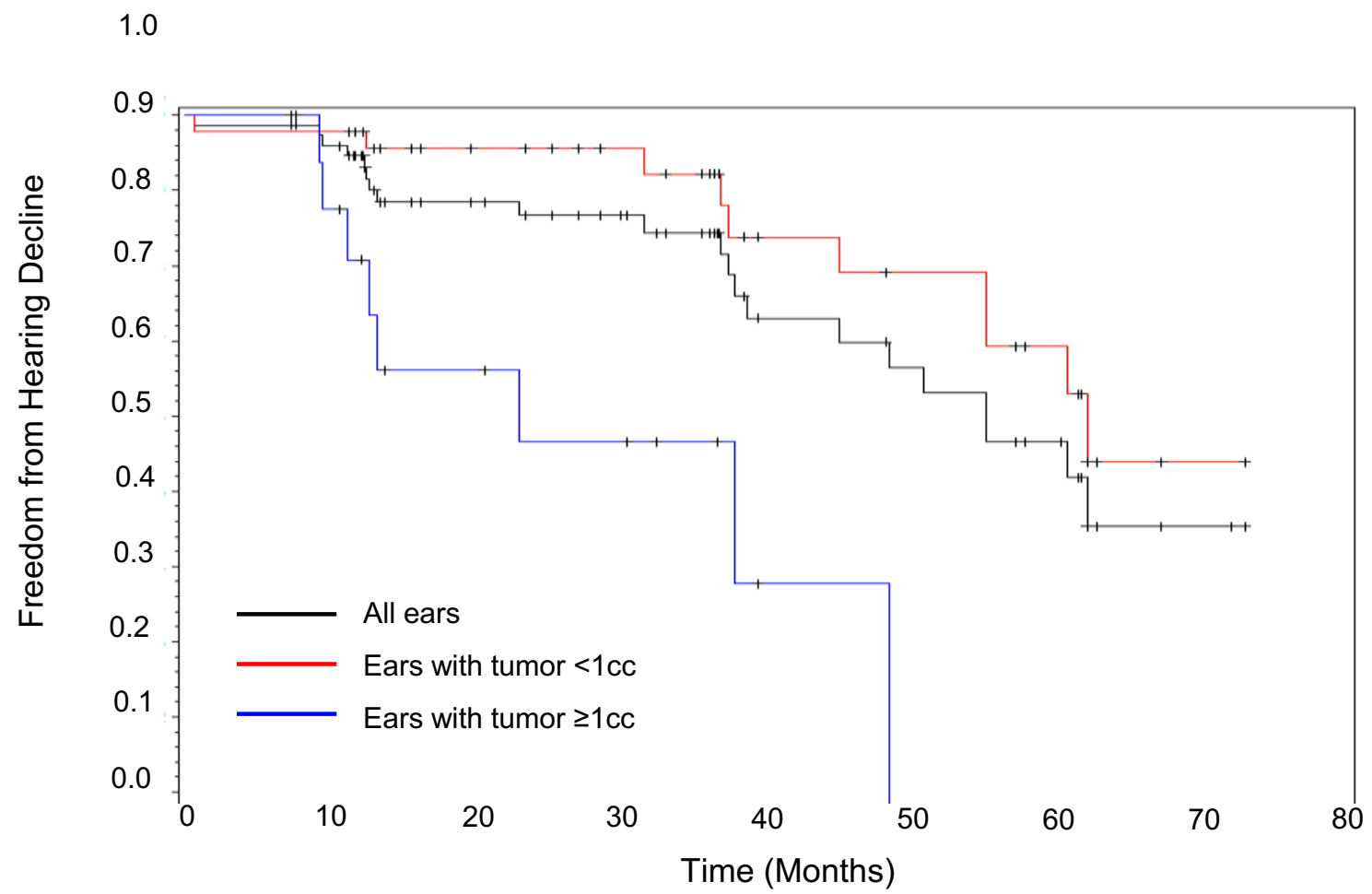

Number at Risk

\begin{tabular}{|c|c|c|c|c|c|c|c|c|}
\hline All tumors & 76 & 71 & 50 & 39 & 23 & 20 & 14 & 2 \\
\hline Tumors < $1 \mathrm{cc}$ & 47 & 45 & 35 & 28 & 18 & 16 & 11 & 1 \\
\hline Tumors $\geq 1 \mathrm{cc}$ & 18 & 15 & 8 & 6 & 1 & 0 & 0 & 0 \\
\hline
\end{tabular}


Figure 3

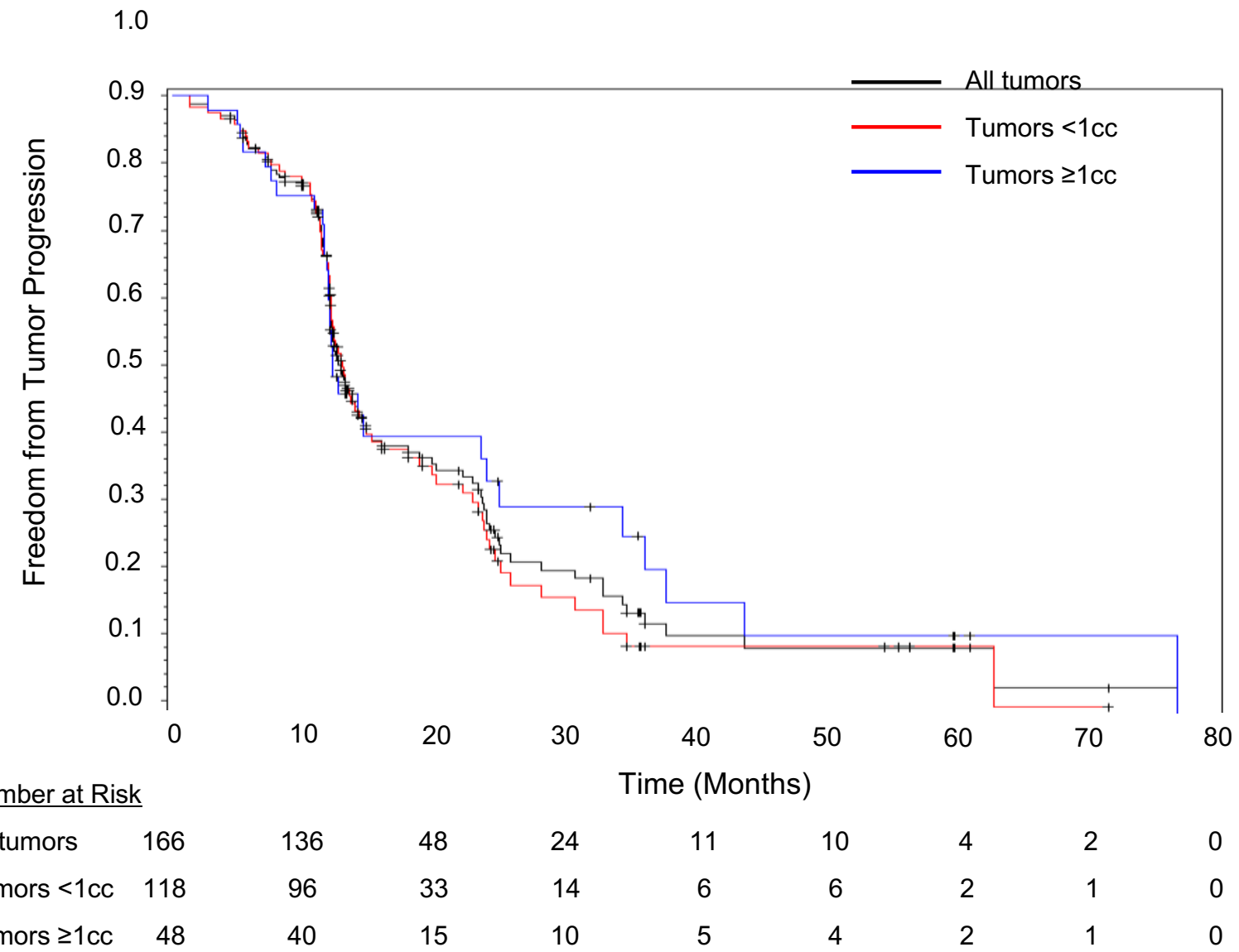


Supplemental Digital Content 1.

Centers participating in the NF2 Natural History Study

- House Ear Institute, Los Angeles, CA, USA (PI: William Slattery, III)

- Hopital Beaujon, Paris, France (PI: Michel Kalamarides)

- Klinikum Nord Ochsenzoll, Hamburg, Germany (PI: Victor Mautner)

- Massachusetts General Hospital, Boston, MA, USA (PI: Mia MacCollin and Scott Plotkin)

- Mt. Sinai School of Medicine, New York, NY, USA (PI: Jeffrey Allen)

- Nagoya University, Nagoya, Japan (PI: Kiyoshi Saito)

- Ohio State University, Columbus, OH, USA (PI: D. Bradley Welling)

- Royal Victorian Eye and Ear Hospital, Melbourne, Australia (PI: Robert Briggs)

- St. Mary's Hospital, London, England (PI: Gareth Evans)

- University of Texas, Houston, TX, USA (PI: Joseph Chang) 
Supplemental Digital Content 2.

Number of patients required for a randomized phase 2 study of NF2 patients with tumor volume

$\geq 1 \mathrm{ml}$, assuming a spontaneous hearing or radiographic response of $15 \%$, a two-sided level of significance of 5\%, and power of $80 \%$ using a two-sided Fisher's Exact test.

\begin{tabular}{|c|c|}
\hline $\begin{array}{c}\text { Estimated drug response rate } \\
\text { (hearing or radiographic) }\end{array}$ & $\begin{array}{c}\text { Total number for } \\
\text { trial }\end{array}$ \\
\hline $20 \%$ & 1812 \\
\hline $30 \%$ & 240 \\
\hline $40 \%$ & 98 \\
\hline $50 \%$ & 54 \\
\hline $60 \%$ & 34 \\
\hline $70 \%$ & 22 \\
\hline $80 \%$ & 16 \\
\hline
\end{tabular}


Supplemental Digital Content 3. Number of patients required for a randomized phase 2 study of NF2 patients using different assumptions for time to progression. All simulations assume a 1year accrual interval, a 3-year follow up period, no trial dropouts, an equal number of patients in each arm, a two-sided level of significance of $5 \%$, and power of $80 \%$.

\begin{tabular}{|c|c|c|c|}
\hline $\begin{array}{c}\text { Minimal } \\
\text { detectable } \\
\text { hazard ratio }\end{array}$ & $\begin{array}{c}\text { Total N for trial (both arms) } \\
\text { assuming median time to } \\
\text { tumor progression of 14.7 } \\
\text { months }\end{array}$ & $\begin{array}{c}\text { Total N for trial (both } \\
\text { arms) assuming median } \\
\text { time to hearing decline } \\
\text { (VS } \geq \mathbf{1} \text { ml) of 38 months }\end{array}$ & $\begin{array}{c}\text { Total N for trial (both } \\
\text { arms) assuming } \\
\text { median time to hearing } \\
\text { decline (all VS) of 62 } \\
\text { months }\end{array}$ \\
\hline 1.25 & 764 & 1278 & 1850 \\
\hline 1.5 & 240 & 418 & 610 \\
\hline 1.75 & 132 & 236 & 346 \\
\hline 2.0 & 90 & 164 & 244 \\
\hline 2.5 & 56 & 106 & 160 \\
\hline 3.0 & 42 & 82 & 124 \\
\hline 4.0 & 30 & 62 & 96 \\
\hline
\end{tabular}

